\title{
TEXTURE CLASSIFICATION BY USING ADVANCED LOCAL BINARY PATTERNS AND SPATIAL DISTRIBUTION OF DOMINANT PATTERNS
}

\author{
Shu Liao and Albert C. S. Chung \\ Lo Kwee-Seong Medical Image Analysis Laboratory \\ Department of Computer Science and Engineering, \\ The Hong Kong University of Science and Technology, Clear Water Bay, Hong Kong. \\ \{liaoshu,achung\}@cse.ust.hk
}

\begin{abstract}
In this paper, we propose a new feature extraction method, which is robust against rotation and histogram equalization for texture classification. To this end, we introduce the concept of Advanced Local Binary Patterns (ALBP), which reflects the local dominant structural characteristics of different kinds of textures. In addition, to extract the global spatial distribution feature of the ALBP patterns, we incooperate ALBP with the Aura Matrix measure as the second layer to analyze texture images. The proposed method has three novel contributions. (a) The proposed ALBP approach captures the most essential local structure characteristics of texture images (i.e. edges, corners); (b) the proposed method extracts global information by using Aura Matrix measure based on the spatial distribution information of the dominant patterns produced by ALBP; and (c) the proposed method is robust to rotation and histogram equalization. The proposed approach has been compared with other widely used texture classification techniques and evaluated by applying classification tests to randomly rotated and histogram equalized images in two different texture databases: Brodatz and CUReT. The experimental results show that the classification accuracy of the proposed method exceeds the ones obtained by other image features.
\end{abstract}

Index Terms - Texture Classification, Advanced Local Binary Patterns, Spatial Distribution

\section{INTRODUCTION}

Analysis of textures plays an important role in many applications in computer vision, for example, image retrieval, face image analysis, and motion analysis. A very challenging problem in texture classification is to extract rotation and histogram equalization invariant features. This problem is also of particular interest because the applications of rotation and histogram equalization sensitive feature extraction methods are strictly limited.

In the past few decades, some researchers have considered applying various kinds of methods to extract rotation invariant texture features. Madiraju et al. [1] extracted rotation invariant features by computing the covariance. Chetverikov used anisotropy features [2] to classify rotated texture images. Also, several rotation invariant feature extraction methods have been proposed by modifying some existing successful rotation sensitive methods. For example, Kashyap and Khotanzad et al. [3] developed an isotropy circular Gaussian MRF (ICGMRF) method [3] based on the GMRF method proposed by Chellappa [4]. Deng and Clausi [5] extended the ICGMRF approach by using similar circular neighborhoods so that it is more robust to non-isotropy textures, which is the anisotropic circular Gaussian MRF model (ACGMRF). Porter and Canagarajah [6] extracted rotation invariant features based on the traditional wavelet transform [7]. They removed the $\mathrm{HH}$ wavelet channels and combined the LH and HL wavelet channels to obtain rotation invariant features. Also, Ojala et al. [8] proposed the uniformed local binary patterns (LBP) approach to extracting rotation and histogram equalization invariant features, which was extended by Huang, Li and Wang by computing the derivative-based local binary patterns and applied it to the application of face alignment [9].

The approach of the conventional LBP is simple and efficient. However, as the conventional LBP just considers the uniform patterns in the images. It discards important pattern information for images whose dominant patterns (e.g. the specific patterns with the largest proportion among all the patterns) are not uniform patterns. Also, the features of the conventional LBP are the histograms of the uniform patterns in a texture image. As such, the spatial distribution information of the patterns (e.g. the locations of the patterns in the image) is lost, which will be shown that it is an important feature to classify the textures in this paper.

Therefore, we are motivated to propose a new rotation and histogram equalization invariant texture classification method by extending the conventional LBP approach to reflect the dominant pattern information contained in the texture images and capturing the spatial distribution information of the dominant patterns. The main contributions of this paper are twofold. First, based on the framework of the conventional LBP, we extend it by observing the frequency histogram of all the la- 
bels of the patterns and then use the histogram distribution of the dominant patterns as features. Therefore, the features of the improved LBP method can describe the dominant pattern structures in a texture image more reliably and effectively.

Second, we find out the locations of all the dominant patterns in a texture image and then construct a binary image for each dominant pattern by marking the locations where such dominant pattern occurs as "1", and "0" otherwise. Therefore, each binary image contains the spatial distribution information of its corresponding dominant pattern. Then, we use the Gray Level Aura Matrix [10] to extract the spatial information features in each binary image. It is found that significant improvement can be made by the modified LBP method. Also, by combining the spatial distribution information of the dominant patterns, further improvement can be achieved. In the experiments, it is observed that high classification accuracy can be achieved even in the tough situation by applying rotation and histogram equalization to the challenging databases of texture images. We utilize the support vector machine [11] as the classifier in this paper. The grid search is performed to find out the best values of the parameters in which the highest classification accuracy can be achieved for each feature.

We evaluate the performance of the proposed approach by performing texture classification experiments in two different databases: Brodatz [12] and CUReT textures [13]. Excellent classification accuracy can be achieved by using the proposed method. This implies that our method can produce robust rotation and histogram equalization invariant features for discriminating a large number of textures. The features of our proposed method are also computationally efficient as it does not need to perform any image filtering.

\section{ADVANCED LOCAL BINARY PATTERNS}

In the conventional LBP approach [8], the image pixels are first labelled by thresholding the difference between the center pixel and its neighbors using the step function $u(x)$ (i.e. $u(x)=1$ when $\mathrm{x} \geq 0$ and $u(x)=0$ otherwise). The concatenation of the neighboring labels is then used as a unique descriptor for each pattern.

The patterns are uniform if the transitions between " 0 " and " 1 " are less than or equal to two. For example, 01100000 and 11011111 are uniform patterns. The histogram of the uniform patterns in the whole image is used as the feature vector [8].

Multiresolution analysis can be achieved by choosing different values of $m$ and $R$, where $m$ denotes the number of neighboring pixels with respect to the center pixel, and $R$ represents the distance from the center pixel to each of the neighboring pixels.

However, for some textures whose dominant patterns are not "uniform local binary patterns", especially for the textures with irregular shapes and edges, the conventional local binary patterns cannot effectively and reliably capture the majority pattern information in the texture images by just considering the "uniform local binary patterns". Table 1 lists the proportions of the "uniform local binary patterns" with different radius $\mathrm{R}$ in some texture images in the CUReT database.

\begin{tabular}{|cccc|}
\hline Textures & $\mathrm{P}=8, \mathrm{R}=1$ & $\mathrm{P}=16, \mathrm{R}=2$ & $\mathrm{P}=24, \mathrm{R}=3$ \\
\hline Loofa0002 & 52.73 & 41.34 & 31.73 \\
Loofa0008 & 57.11 & 45.73 & 38.10 \\
Brown Bread0017 & 53.18 & 39.95 & 24.82 \\
Brown Bread0022 & 47.69 & 32.42 & 20.76 \\
Concrete 0009 & 52.83 & 44.04 & 35.18 \\
Concrete 0016 & 47.91 & 36.04 & 26.17 \\
Crumpled Paper0015 & 50.05 & 46.18 & 33.06 \\
Crumpled Paper0016 & 57.85 & 48.82 & 36.11 \\
\hline
\end{tabular}

Table 1. Proportions (\%) of "uniform LBP" for some samples in the CUReT database. It shows that, for some kinds of textures, the dominant patterns are not mainly the "uniform LBP".

In order to fully describe the dominant patterns contained in the texture images, we extend the conventional LBP to ALBP. Each pattern in the image is assigned a unique label by the following equation [8]

$$
\operatorname{LBP}(m, R)=\sum_{i=0}^{p-1} u\left(t_{i}-t_{c}\right) 2^{i},
$$

where $t_{c}$ is the intensity of the center pixel, $t_{i}$ is the intensity of the neighbor $i$, and $u(x)$ is the step function. It is obvious that the LBP defined in Equation 1 is not rotation-invariant as the intensity value of $t_{i}$ changes when the circle is rotated by a specific angle. Two patterns should be treated as the same type if one can be obtained from the other through rotating by a certain angle. Therefore, the ALBP pattern group is defined as,

$$
A L B P^{s}(m, R)=\min (C i r(L B P(m, R), n)),
$$

where $n=0,1, \ldots, p-1, \operatorname{Cir}(x, n)$ performs a circular anticlockwise bitwise shift on the $p$-bit number by $n$ times.

The histogram of the ALBP group is computed from the image and sorted in a descending order. Therefore, the first several entries of the histogram are guaranteed to be the dominant patterns from the image. Our experiments show that around $80 \%$ of the patterns in the image are sufficient for representing the information of the dominant patterns, and the 20 leading entries are enough to cover $80 \%$ of the whole ALBP histogram in the texture image.

\section{SPATIAL DISTRIBUTION INFORMATION OF DOMINANT PATTERNS}

Although the advanced local binary patterns can more reliably and effectively describe the dominant pattern information and more robust to random rotation, the spatial distribution information of the dominant patterns (SIDP) is still lost. More precisely, by using the ALBP alone, we only know what are the dominant patterns in a texture image. However, we do not know where are the locations of such dominant patterns. 

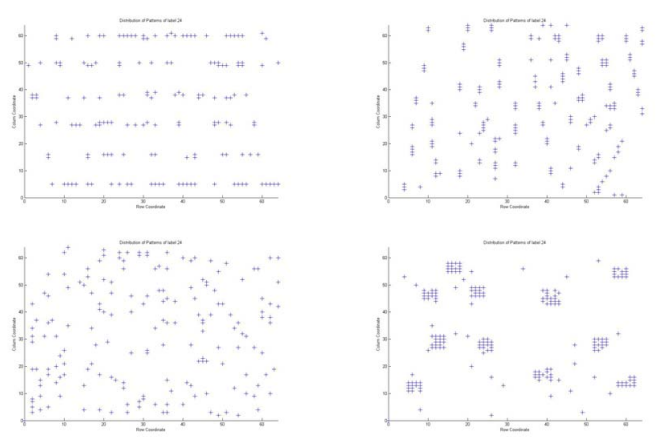

Fig. 1. Spatial distribution maps of dominant pattern labelled 24 in C32, C34, C14 and C17.
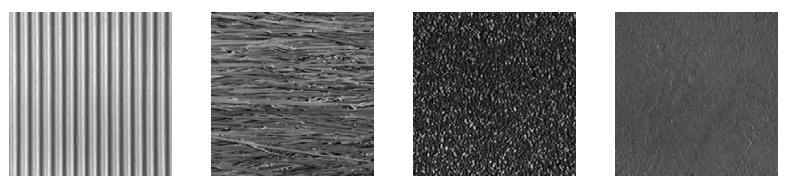

Fig. 2. The four textures obtained from the CUReT database. From left to right: C32, C34, C14, C17.

Such information actually is a powerful feature to describe the texture image. To illustrate the concepts of SIDP, Fig. 1 plots the distribution of a dominant pattern in several texture images obtained from the Curet [13] database.

The four texture images in Fig. 2 actually are misclassified by using only the conventional LBP or ALBP according to our experimental results. The reason is that although ALBP can describe the types of dominant patterns in the texture more reliably, the spatial distribution information of such patterns is lost. In the above four textures, they all have the same sets of dominant patterns with very similar proportions, and the pattern type labelled with 24 is one of the dominant patterns. As we can see, the numbers of such dominant patterns in each texture are very close to each other. However, the distribution properties of this pattern in these four texture images are very different with each other. Therefore, the SIDP is a very important property to describe the textures, which will be further illustrated in the Experimental Results section.

To extract features from the each dominant pattern distribution maps, we use the Gray Level Aura Matrix (GLAM) approach [10] to extract features from the dominant pattern distribution maps. Since now the input image is the dominant pattern distribution maps, which can be treated as a binary image with only two gray levels (with value " 1 " assign to the locations where they contain such pattern, and "0" otherwise), it is very efficient to calculate the GLAM from this binary image with only two gray levels. There are only four elements in the resulting GLAM. Then, these four elements are used as features to describe the spatial distribution characteristic of the dominant patterns.

Some basic concepts related to the GLAM [10] are now introduced. Suppose that we represent an image $X$ with a rectangular lattice of $m \times n$ grids $S$, where $m$ and $n$ denote the width and height of $X . X$ now can be represented by $\mathrm{S}$ as,

$$
S=\{s=(i, j) \mid 0 \leq i \leq m-1,0 \leq j \leq n-1\} .
$$
Also, a neighborhood system $N=\left\{N_{s}, s \in S\right\}$ is defined, where $N_{s}$ is the neighborhood at site s. There are two important properties in the neighborhood system $\mathrm{N}$ : (a) $\mathrm{s} \notin N_{s}$ and (b) $\mathrm{s} \in N_{t}$ if an only if $\mathrm{t} \in N_{s}$. Property (a) implies that site $\mathrm{s}$ is not included in its neighborhood. Property (B) implies that the neighborhood is symmetric. In this paper, we use a symmetric $11 \times 11$ window with the target pixel at the center as the neighborhood system.

Aura: [10] Given two subsets $A, B \subseteq S$, the aura of $A$ with respect to $B$ for neighborhood system $N$, denoted $\vartheta_{B}(A, N)$, is given by,

$$
\vartheta_{B}(A, N)=\cup_{s \in A}\left(B \cap N_{s}\right) .
$$

Aura Measure: [10] With the same notations as in Eq. 4, the aura measure of $A$ with respect to $B$, denoted by $m(A, B)$, is given by:

$$
m(A, B)=\sum_{s \in A}\left|N_{s} \cap B\right|,
$$

where for a given subset $A \subseteq S,|A|$ is the total number of elements in $A$.

Gray Level Aura Matrix (GLAM): [10] Let $N$ be the neighborhood system over $S$, and $\left\{S_{i}, 0 \leq i \leq G-1\right\}$ be the gray level sets of an image over $S$. Then, the gray level aura matrix of the image over $N$, denoted $A$, is given by,

$$
A=A(N)=\left[m\left(S_{i}, S_{j}\right)\right],
$$

where $G$ is the total number of gray levels in the image, $S_{i}=$ $\left\{s \in S \mid x_{s}=\mathrm{i}\right\}$ is the gray level set corresponding to the $i^{\text {th }}$ level, and $m\left(S_{i}, S_{j}\right)$ is the aura measure between $S_{i}$ and $S_{j}$ defined in Eq. 5.

The resulting four elements in the GLAM are strong features, which can describe the spatial distribution information of the dominant patterns and are used as features to complement with the ALBP.

\section{EXPERIMENTAL RESULTS}

We have evaluated our proposed method on two different databases with large sets of texture images: (1) 24 textures selected from the Brodatz album [12]; (2) 47 textures selected from the CUReT database [13]. In our experiment, random rotation (each image in the training set and testing set was rotated by a randomly generated angle between 0 to 360 degrees) and histogram equalization were performed on each sample on both the training and testing sets in order to test the robustness of method. Half of the images in each class were used as training sets and the remaining images were used as testing sets. Our approach has been compared with eight widely used texture 
classification methods. In our experiment, the support vector machine (SVM) was used as the classifier. The kernel for the SVM was the Gaussian Radial Basis Function (RBF).

Experiments on Brodatz Database: The classification accuracies of different approaches under different environments are listed in Table 2. According to the experimental results, the proposed ALBP approach can already outperform the other eight methods under different conditions. Also, by embedding the spatial distribution information of dominant patterns (SIDP) with the ALBP, the classification performance is better than using ALBP alone.

Experiments on CUReT Database: In the CUReT database [13], it has the largest number of texture classes (47 classes). The major characteristic of this database is that the number of texture classes is very large. Therefore, it is very difficult to classify such large number of textures because it can have small inter-class distances in the feature space. This database actually can test how precise can the features of each approach describe the texture images. The experimental results are listed in Table 3. We can see that, by embedding the SIDP features to the ALBP features, our proposed method gives very good performance.

\begin{tabular}{|c|c|c|c|c|}
\hline \multirow[b]{2}{*}{ Features } & \multicolumn{4}{|c|}{ Classification accuracy \% } \\
\hline & $\begin{array}{l}\text { Original } \\
\text { Textures }\end{array}$ & $\begin{array}{c}\text { Histogram } \\
\text { Equalized } \\
\text { Textures }\end{array}$ & $\begin{array}{l}\text { Randomly } \\
\text { Rotated } \\
\text { Textures }\end{array}$ & $\begin{array}{l}\text { Histogram Equalized \& } \\
\text { Random Rotated }\end{array}$ \\
\hline DBWP [7] & 98.06 & 87.73 & 81.32 & 62.71 \\
\hline RDBWP [6] & 91.67 & 75.00 & 91.20 & 76.83 \\
\hline TGF [14] & 98.61 & 91.67 & 83.51 & 61.36 \\
\hline CGF [14] & 90.07 & 60.28 & 88.64 & 58.42 \\
\hline GMRF [4] & 96.70 & 84.33 & 50.63 & 40.75 \\
\hline ACGMRF [5] & 95.83 & 86.52 & 93.75 & 81.65 \\
\hline MRH [15] & 93.57 & 70.00 & 87.35 & 58.31 \\
\hline LBP [8] & 97.22 & 96.30 & 92.75 & 91.50 \\
\hline ALBP & 98.61 & 98.61 & 96.78 & 96.76 \\
\hline ALBP with SIDP & 99.85 & 99.54 & 99.54 & 99.54 \\
\hline
\end{tabular}

Table 2. Performance of different features in the Brodatz Database. Results of our methods are listed in the last two rows. For each test (column), the highest classification accuracy is highlighted in bold. DBWP: Daubechies wavelet packet features; RDBWP: Rotational invariant DBWP; TGF: Traditional Gabor filters; CGF: Circular Gabor filters; GMRF: Gaussian Markov random fields; ACGMRF: Anisotropic circular Gaussian MRFs; MRH: Mutiresolution histograms; LBP: Uniform local binary patterns; ALBP: Advanced local binary patterns; SIDP: Spatial Information of Dominant Patterns.

\begin{tabular}{|c|cccc|}
\hline & \multicolumn{4}{|c|}{ Classification accuracy \% } \\
& $\begin{array}{c}\text { Original } \\
\text { Features }\end{array}$ & $\begin{array}{c}\text { Histogram } \\
\text { Equalized } \\
\text { Textures }\end{array}$ & $\begin{array}{c}\text { Randomly } \\
\text { Rotated } \\
\text { Textures }\end{array}$ & $\begin{array}{c}\text { Histogram Equalized \& } \\
\text { Random Rotated } \\
\text { Textures }\end{array}$ \\
\hline DBWP [7] & 90.43 & 61.17 & 80.13 & 54.46 \\
RDBWP [6] & 85.11 & 39.89 & 83.75 & 45.81 \\
TGF [14] & 76.06 & 44.15 & 63.25 & 33.56 \\
CGF [14] & 60.64 & 27.66 & 61.53 & 36.80 \\
GMRF [4] & 46.81 & 45.27 & 27.04 & 35.63 \\
ACGMRF [5] & 77.13 & 71.26 & 76.59 & 68.25 \\
MRH [15] & 69.15 & 38.83 & 63.68 & 38.21 \\
LBP [8] & 72.87 & 69.15 & 70.25 & 62.58 \\
\hline ALBP & 82.98 & 79.79 & 84.18 & 76.02 \\
ALBP with SIDP & $\mathbf{9 7 . 6 4}$ & $\mathbf{9 6 . 5 7}$ & $\mathbf{9 6 . 5 7}$ & $\mathbf{9 5 . 8 0}$ \\
\hline
\end{tabular}

Table 3. Performance of different features of $64 \times 64$ image resolution in the CUReT Database. Results of our methods are listed in the last two rows. For each test (column), the highest classification accuracy is highlighted in bold.

\section{CONCLUSION}

In this paper, we have proposed a new advanced local binary pattern approach based on the conventional LBP. Also, we find that the spatial distribution information of dominant patterns (SIDP) actually is a very powerful feature for describing the characteristics of the texture image as it includes the location information of the dominant patterns in the texture images. It has been evaluated by comparing with eight widely used approaches with two databases: Brodatz and CUReT. It is experimentally shown that our approach has excellent performance in texture classification and is very robust to histogram equalization and random rotation. Computational simplicity is another advantage of our proposed method as the features can be obtained with only a few calculations and comparisons without the need of performing any image filtering.

\section{REFERENCES}

[1] S.V.R. Madiraju and C.C Liu, "Rotation invariant texture classification using covariance," in Proc. Int'l Conf. Image Processing, 1994, vol. 2, pp. 655-659.

[2] D. Chetverikov, "Experiments in the rotation-invariant texture discrimination using anisotropy features," in Proc. Sixth Int'l Conf. Pattern Recognition, 1982, vol. 18, pp. 975-985.

[3] R.L. Kashyap and A. Khotanzad, "A model-based method for rotation invariant texture classification,” PAMI, vol. 8, no. 7, pp. 472-481, 1986.

[4] R. Chellappa and S. Chatterjee, "Classification of textures using gaussian markov random fields," IEEE Trans. ASSP, vol. 33, no. 4, pp. 959-963, 1985.

[5] D.A Huawu Deng, Clausi, "Gaussian mrf rotation-invariant features for image classification,” PAMI, vol. 26, no. 7, pp. 951-955, 2004.

[6] R. Porter and N. Canagarajah, "Robust rotation-invariant texture classification: Wavelet, gabor filter and gmrf based schemes," in IEE Proc. Conf. Vision, Image, and Signal Processing, 1997, vol. 144, pp. 180 188.

[7] A. Laine and J. Fan, "Texture classification by wavelet packet signatures," PAMI, vol. 15, no. 11, pp. 1186-1191, 1993.

[8] M. Pietikainen T. Ojala and T. Maenpaa, "Multiresolution gray-scale and rotation invariant texture classification with local binary patterns," PAMI, vol. 24, no. 7, pp. 971-987, 2002.

[9] S.Z.Li X. Huang and Y. Wang, "Shape localization based on statistical method using extended local binary pattern," in IEEE Proc. Conf. Image and Graphics, 2004, pp. 184-187.

[10] Xuejie Qin and Yee-Hong Yang, "Similarity measure and learning with gray level aura matrices (glam) for texture image retrieval," in CVPR, 2004, vol. 1, pp. 326-333.

[11] V.N. Vapnik, Ed., Statistical Learning Theory, John Wiley and Sons, New York, 1998.

[12] P. Brodatz, "Textures: A photographic album for artists and designers," in Dover, 1966.

[13] S. Nayar K. Dana, B. Ginneken and J. Koenderink, "Reflectance and texture of real-world surfaces," ACM Trans. Graphics, vol. 18, no. 1, pp. 1-34, 1999.

[14] G.M. Haley and B.S. Manjunath, "Rotation-invariant texture classification using a complete space-frequency model," IEEE Trans. Img. Proc., vol. 8, no. 2, pp. 255-269, 1999.

[15] D. Michael H. Efstathios and S. Nayar, "Multiresolution histograms and their use for recognition," PAMI, vol. 26, no. 7, pp. 831-847, 2004. 